\title{
Optical Properties of CdTe QDs Formed Using Zn Induced Reorganization
}

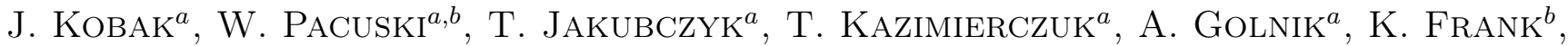 \\ A. Rosenauer ${ }^{b}$, C. $\mathrm{KRuSE}^{b}$, D. Hommel ${ }^{b}$ And J.A. Gaj ${ }^{a}$ \\ ${ }^{a}$ Institute of Experimental Physics, Faculty of Physics, University of Warsaw, Hoża 69, PL-00-681 Warszawa, Poland \\ ${ }^{b}$ Institute of Solid State Physics, University of Bremen, P.O. Box 330 440, D-28334 Bremen, Germany \\ In this paper we present optical studies of CdTe quantum dots formed using Zn-induced reorganization. The \\ pattern of quantum dot photoluminescence lines is found to be similar to typical results reported for quantum \\ dots grown with other techniques, although the positively charged exciton line is relatively more pronounced. Also \\ the energy spacing between biexciton and exciton lines is found to be larger than in typical results. Zn-induced \\ reorganization results in quantum dots density higher by an order of magnitude than in Te-induced quantum dots.
}

PACS: 81.07.Ta, 78.67.Hc

\section{Introduction}

Strain induced formation of quantum dots (QDs) works very well for structures based on III-V compounds, such as InAs/GaAs QDs. For II-VI materials, strain is usually not sufficient to induce the Stranski-Krastanov formation of QDs, therefore an additional treatment is necessary. This work is devoted to the influence of growth conditions on optical properties of self-assembled CdTe/ ZnTe QDs.

\section{Sample and experiment}

The growth of the structures was performed by molecular beam epitaxy (MBE) on GaAs substrate. First, a $1 \mu \mathrm{m}$ thick ZnTe buffer layer has been deposited. It was found to be fully relaxed according to measurements of high-resolution X-ray diffraction (HRXRD). Next, a CdTe layer was deposited using migration enhanced epitaxy (MEE), i.e. the alternating supply of $\mathrm{Cd}$ and Te interrupted by a pause. In each cycle, about 0.2 of monolayer was deposited. Total thickness of CdTe layer was determined from in situ reflectivity measurements. Then, a ZnTe barrier was deposited either directly, or preceded by one of two reorganization methods: well established amorphous Te desorption [1, 2], or our novel method - Zn-induced reorganization of CdTe (MEE growth of Zn-rich ZnTe). In contrast to the method with Te desorption, Zn-induced reorganization did not require temperature ramp. Scanning transmission electron microscopy (STEM) reveals a typical lateral size of 5-10 nm and a height of $2 \mathrm{~nm}$ for the CdTe QDs grown using Zn-induced reorganization. The $\mathrm{Zn}$-induced reorganization had been previously applied in fabrication of CdSe QDs in ZnSe matrices [3]. In order to deposit Zn-rich layer, we applied $10 \mathrm{MEE}$ cycles of $\mathrm{Zn}$ and Te. In each cycle there was 5 or $15 \mathrm{~s}$ of $\mathrm{Zn}$ flux and $5 \mathrm{~s}$ of Te flux.
In our investigations we used polarization-resolved macro- and microphotoluminescence. In microphotoluminescence, the sample was immersed in superfluid helium together with a micro-lens $(f=3 \mathrm{~mm})$, which made possible to resolve the luminescence from single QDs. The micro-lens was controlled by three piezo-step translation stages in all three perpendicular directions $(x y z)$. An Ar ion laser (488 nm) or a solid state green laser (532 nm) were used to excite the samples. The intensity of both lasers was varied independently using neutral density filters.

\section{Macrophotoluminescence}

Results of macro-PL measurements show high intensity emission for QDs grown using Zn-induced reorganization, weaker PL of QDs grown using Te induced reorganization (Fig. 2), and very weak PL of CdTe layer covered by usual ZnTe (not shown).

A red shift is observed (Fig. 2) with increasing amount of Zn. For Zn-induced QDs no wetting layer is observed, contrary to Te-induced reorganization, for which wetting layer emission is observed, in contrast with previous reports [4].

For samples with Zn-induced QDs a red shift and a broadening with increasing CdTe layer thickness are observed (Fig. 3, Fig. 4). The thickness of CdTe layer was determined from in situ reflectivity during the growth of the sample.

\section{Microphotoluminescence}

Micro-PL studies confirm the presence of sharp QD lines for all growth techniques (Fig. 5). For all samples we find a characteristic pattern of single QD emission (Fig. 5), however, relative intensities of various excitonic lines are different from those typically observed. The line 


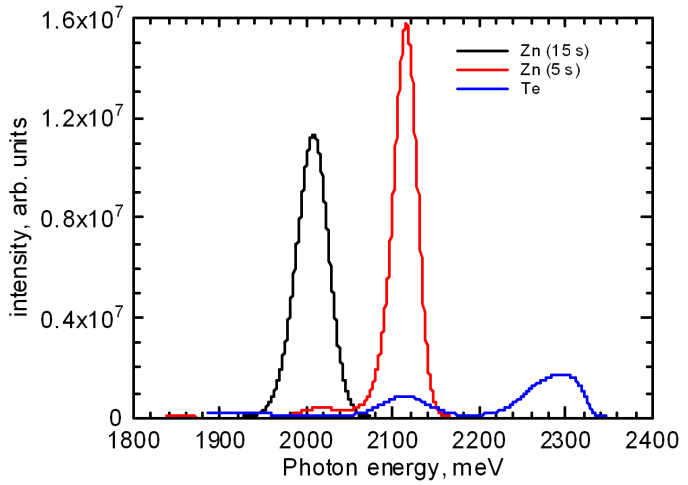

Fig. 1. Macro-PL of three samples grown using different methods. Thickness of CdTe formation layer is the same for all the samples $(d=2 \mathrm{~nm})$.

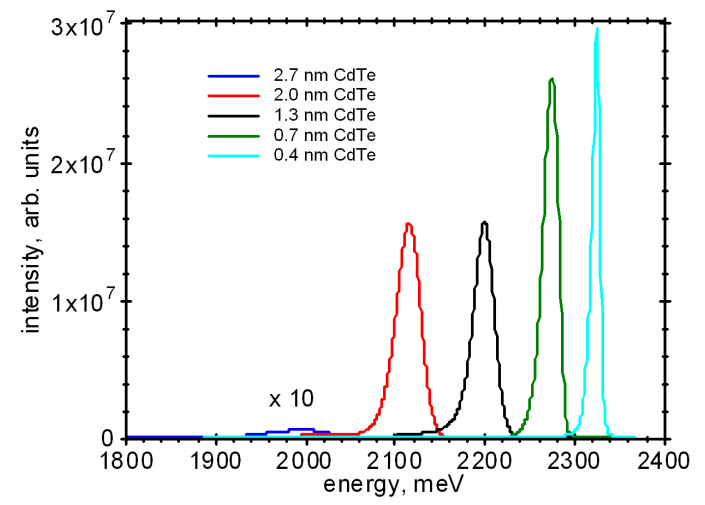

Fig. 2. Macro-PL of samples with different thickness of CdTe layer.

identified as negatively charged exciton $\left(\mathrm{X}^{+}\right)$is the most intense, in contrast to the exciton $(\mathrm{X})$ line, which is very weak.

The energy spacing between the exciton $(\mathrm{X})$ and biexciton (XX) lines is about $15 \mathrm{meV}$, which is a little bit

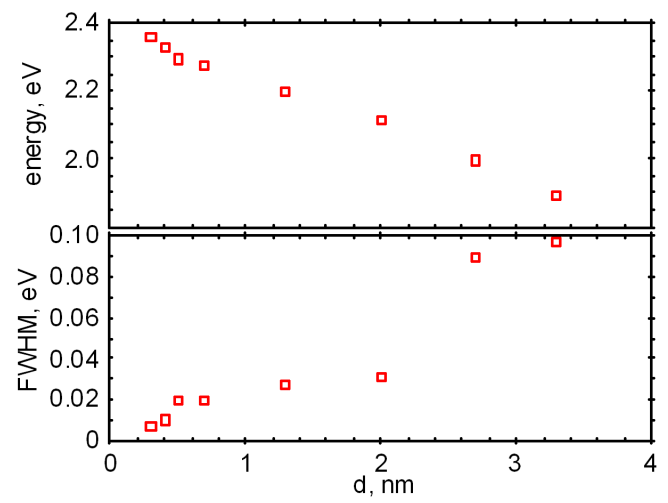

Fig. 3. Emission energy and peak linewidth determined for samples with different thickness of CdTe layer.

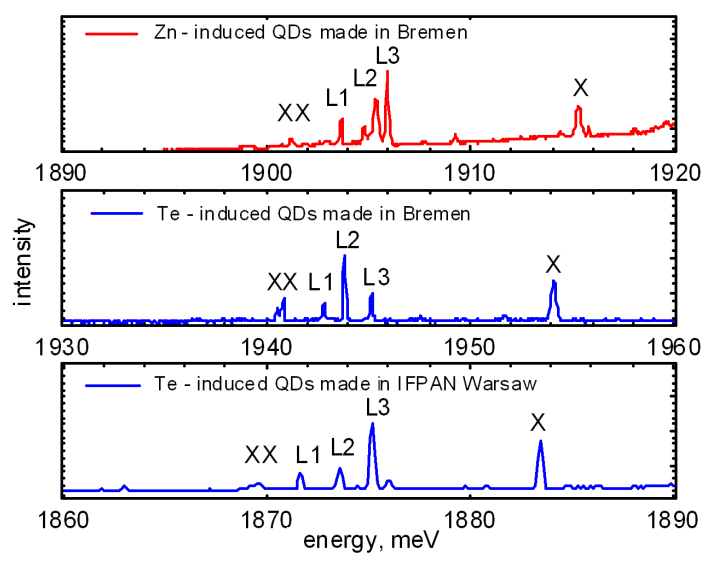

Fig. 4. Micro-PL at liquid helium temperature. Characteristic pattern of single QDs emission for samples grown using various methods.

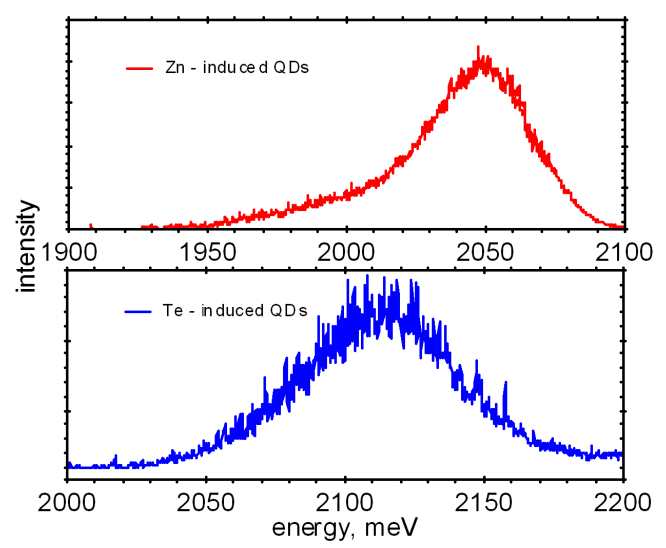

Fig. 5. Micro-PL showing impact of growth technique on QDs density.

greater than typical results $(13 \mathrm{meV})$ known for CdTe/ ZnTe QDs [1]. Independently of the growth technique, experimental results show that there is no correlation between biexciton-exciton energy spacing and exciton energy (Fig. 6).

The largest QDs density is obtained for QDs induced with Zn, smaller for QDs induced with Te, and the smallest for CdTe layer covered directly with ZnTe. Zn-induced reorganization results in QDs density higher by an order of magnitude than density of QDs obtained by Te-induced reorganization (Fig. 7).

For both $\mathrm{Zn}$ and Te-induced QDs we determine position of $\mathrm{QD}$ photoluminescence lines with respect to $\mathrm{X}$ line vs. XX-X energy spacing (Figs. 8 and 9 ). As a result we establish that while the energy spacing between $\mathrm{XX}$ and $\mathrm{X}$ lines varies significantly from dot to dot, the relative position of $\mathrm{QD}$ photoluminescence lines is constant. The results obtained for Zn-induced QDs are very similar to those obtained for Te-induced QDs.

Summarizing, Zn-induced reorganization has been shown to be an efficient method of $\mathrm{CdTe} / \mathrm{ZnTe} \mathrm{QD}$ 


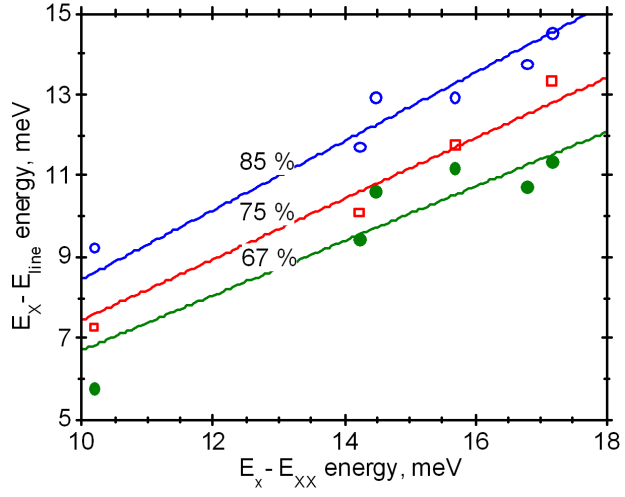

Fig. 6. Energy position of QD PL lines with respect to $\mathrm{X}$ line vs. $\mathrm{XX}-\mathrm{X}$ energy spacing - $\mathrm{Zn}$ induced QDs. Numbers indicate lines slopes.

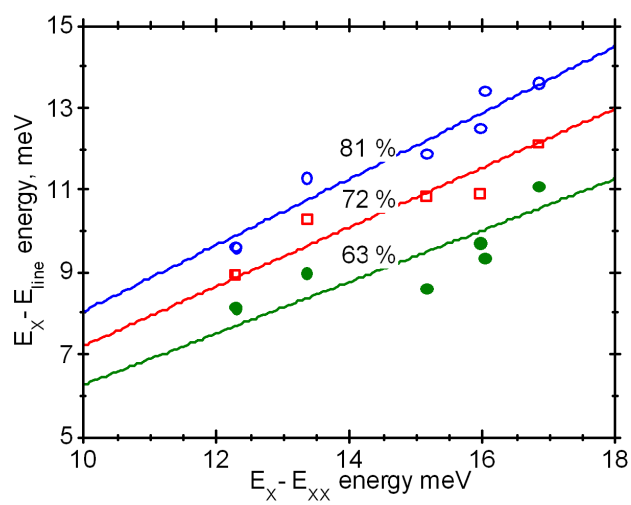

Fig. 7. Energy position of QD PL lines with respect to $\mathrm{X}$ line vs. XX-X energy spacing - Te induced QDs. Numbers indicate lines slopes. growth. The fabricated QDs exhibit typical densities higher from Te-induced QDs by an order of magnitude and good luminescent properties. The observed typical pattern of PL lines of a single QD is identical to that known for QDs grown by tellurium desorption technique.

\section{Acknowledgments}

This work was supported by Polish public funds in years 2010-2011 (project Iuventus Plus).

\section{References}

[1] T. Kazimierczuk, J. Suffczyński, A. Golnik, J. Gaj, P. Kossacki, P. Wojnar, Phys. Rev. B 79, 153301 (2009).

[2] F. Tinjod, B. Gilles, S. Moehl, K. Kheng, H. Mariette, Appl. Phys. Lett. 82, 4340 (2003).

[3] C. Kruse, M. Gartner, A. Gust, D. Hommel, Appl. Phys. Lett. 90, 221102 (2007).

[4] F. Tinjod, S. Moehl, K. Kheng, B. Gilles, H. Mariette, J. Appl. Phys. 95, 102 (2004). 